\title{
Erratum to: Decay Rates to Equilibrium for Nonlinear Plate Equations with Degenerate, Geometrically-Constrained Damping
}

\author{
Pelin G. Geredeli • Justin T. Webster
}

Published online: 11 October 2014

(C) Springer Science+Business Media New York 2014

\section{Erratum to: Appl Math Optim (2013) 68: 361-390 DOI 10.1007/s00245-013-9210-8}

It was brought to our attention that line 15 on page 374-which claims $u_{t}=0$ in $\Omega \times \Theta$-is unclear. Upon further inspection, this statement should differentiate between two cases: (a) $p(\mathbf{x})=0$ on $\Omega$ and (b) $p(\mathbf{x}) \neq 0$ (taken in the $L_{2}(\Omega)$ sense). Case (a): As argued in the treatment, the application of Holmgren's theorem gives $u=0$ on $\omega \times \Theta$. The desired final conclusion follows by inserting this information into Berger's equation (by assumption, no longer containing the damping term $d(\mathbf{x}) g\left(u_{t}\right)$ ), and then employing Kim's unique continuation result Theorem 3.1. Case (b): One reaches a contradiction with the additional mild assumption that there exists a set of positive measure $U \subset \omega$ so that $p(\mathbf{x}) \neq 0$ on $U$. Thus Theorem 3.2, and consequently Theorems 2.5, 2.6 (Main Results), remain valid when (a) $p(\mathbf{x}) \equiv 0$, and when (b) $p(\mathbf{x}) \in L_{2}(\Omega)$ is non-trivial, assuming that $p(\mathbf{x}) \neq 0$ on some open set $U \subset \omega$.

Remark 0.1 If $p(\mathbf{x})$ has sufficient regularity then, via the bootstrapping procedure in the proof of Lemma 3.4, the equality

$$
u_{t t}+\Delta^{2} u=f_{B}(u)+p(\mathbf{x})
$$

The online version of the original article can be found under doi:10.1007/s00245-013-9210-8.

\section{P. G. Geredeli}

Hacettepe University, Ankara, Turkey

e-mail:pguven@hacettepe.edu.tr

J. T. Webster $(\varangle)$

Oregon State University, Corvallis, OR, USA

e-mail: websterj@math.oregonstate.edu 
holds pointwisely; by inserting $u \equiv 0$ on $\omega \times \Theta$ we may reach a contradiction to case (b) (as above) if there is a single point $\mathbf{x}_{0} \subset \omega$ such that $p\left(\mathbf{x}_{0}\right) \neq 0$.

We also note that Kim's theorem is more robust: $\mathrm{p}(\mathbf{x})$ may be replaced by $p(u)$ (under suitable dissipativity assumptions); in this case the above proof of the unique continuation result will remain valid. 